\title{
Use of Intragastral Balloon and Increase in Fertility:
}

\section{Case Report}

Leite Letícia Torres ${ }^{1}$, Araújo Anna Bárbara Ribeiro ${ }^{1}$, Souza Luma Lorraine dos Reis ${ }^{1}$, Sander Bruno Queiroz ${ }^{2}$ and Lopes Joanilva Ribeiro ${ }^{3}$

1. Medical school of FAMINAS-BH, College of Minas-BH, Belo Horizonte 30110012, Brazil

2. Bariatric Endoscopy by UFMG, Belo Horizonte 30150285, Brazil

3. UNIMONTES and Prominas Colleges, Montes Claros 39403440, Brazil

\begin{abstract}
The IGB (intragastric balloon) is a silicone device, inflated by endoscopy in the gastric cavity, with physiological saline and a blue dye, up to the volume of $700 \mathrm{~mL}$, and used for a maximum of 6 months. It is indicated for patients with BMI $\geq 27 \mathrm{~kg} / \mathrm{m}^{2}$ that have not undergone previous gastric surgery. In obese women there is a decrease in peripheral resistance fertility, and the weight loss achieved in bariatric surgery improves fertility due to reduced subcutaneous fat, with lower insulin resistance. Similar results could be achieved through the use of IGB, a less invasive method. The objective of the article is to report the case of a patient who became pregnant using IGB, considering the hypothesis that the increase in fertility was due to weight loss and/or due to the improvement in the self-esteem (and sexual life) of patient. We selected 13 articles from PubMed, UpToDate, JAMA and a case report. Results: Balloon with drawal occurred on 05/02/16 after positive pregnancy test with final weight $65.5 \mathrm{~kg}$ and BMI $25.91 \mathrm{~kg} / \mathrm{m}^{2}$. Conclusions: Weight loss actually favors fertility, but self-esteem may have been instrumental in improving the sex life, enabling conception.
\end{abstract}

Key words: IGB, fertility, pregnancy, obesity.

\section{Introduction}

Introduced in 1985, placement of the intragastric balloon is a procedure that restricts the volume of the stomach. It is a procedure that requires a slight sedation and its insertion is done through endoscopy. Immediately after introduction, the flask is inflated with about 500 to $700 \mathrm{~mL}$ of saline stained with methylene blue dye for the purpose of reducing stomach capacity and food intake as shown in Fig. 1 [1]. Currently, three intragastric balloon devices are approved by the FDA in the United States: Orbera Intragas Balloon System (Apollo Endosurgery Inc., Austin, Texas, United States), ReShape ${ }^{\circledR}$ Integrated Dual Balloon System (ReShape Medical, Inc., San Clemente, HERE, United States), and Obalon (Obalon Therapeutics, Inc.). These devices are indicated for patients with Class 1 and 2 obesity (BMI 30-40 kg/m²)

Corresponding author: Anna Bárbara Ribeiro Araujo, physician, research fields: pregnancy and obesity.
[2].

Obese women of reproductive age are a specific risk group for obesity related to infertility and obstetric complications. Infertility is defined as the inability of a couple to achieve pregnancy after 1 year of unprotected regular intercourse or the inability of a woman to conduct a pregnancy until the birth of a living being or with no assisted reproductive technology. There is a strong association between obesity and infertility, and weight loss may result in increased fecundity in obese women [3].

In this context, health is a relevant determinant of the quality of life of a person, and this perception can be affected by aspects related to the culture, religion, environment and education of the individual. In this way, HRQOL (health-related quality of life) is becoming an important indicator of health outcomes. Different studies have shown the relation of overweight and poor health-related quality of life. Pathological conditions related to obesity may contribute 


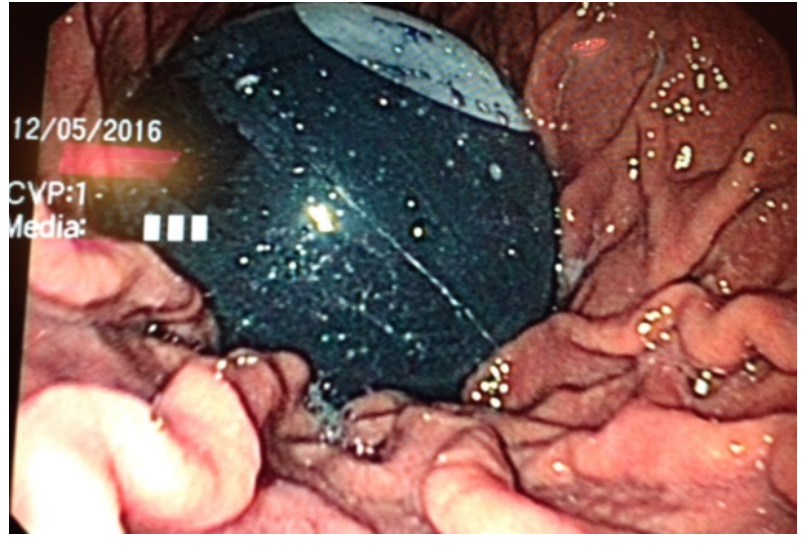

Fig. 1 Clínica Sander.

to HRQOL deficiency in obese people. Different social and psychological aspects may also contribute to worse quality of life in overweight patients, such as criticism, feelings of inferiority, low self-esteem, anxiety and depression. Different weight loss strategies are not only associated with clinical and psychological benefits, but also with a better perception of quality of life. The IGB (intragastric balloon) is one of the strategies currently available for weight loss with significant results in a short period of time [4].

\section{Objective}

The objective of the present article is to report the case of a patient who became pregnant using IGB, considering the hypothesis that increased fertility was due to weight loss and/or due to improved self-esteem (and sexual life) of the patient. We selected 13 articles from PubMed, UpToDate, JAMA and a case report.

\section{Materials and Methods}

This is the case of a 38 years old female patient, married, teacher, native of Belo Horizonte, initial weight of $80 \mathrm{~kg}$ and BMI (Body Mass Index) $31.34 \mathrm{Kg} / \mathrm{m}^{2}$. She was on hormonal treatment for infertility and for obstetrical recommendations she placed her first BIG on 08/02/2013 in order to lose weight and thus become pregnant. She retired from BIG on $8 / 25 / 2013$, when he discovered his first pregnancy, with a final weight of $68.7 \mathrm{~kg}$ and a BMI of
$21.17 \mathrm{~kg} / \mathrm{m}^{2}$. On $10 / 14 / 15$, with $86 \mathrm{~kg}$ and BMI of $34.02 \mathrm{~kg} / \mathrm{m}^{2}$, decided to place the second BIG due to weight gain of the first gestation. During this time, the patient reported weakness and difficulty feeding, which worried her, because she was breastfeeding her son already 8 months. I was not using any contraceptive method. In February 2016, she discovered she was pregnant with her second child, when it was necessary to remove the BIG again, with a final weight of $65.5 \mathrm{~kg}$ and a BMI of $25.92 \mathrm{~kg} / \mathrm{m}^{2}$. After a withdrawal of the second BIG, the patient is not continued with nutritional and psychological monitoring, so in April 2016 its weight was already $75.5 \mathrm{~kg}$, and in September 2016, with 39 weeks of gestation, its weight was $89 \mathrm{~kg}$. One patient reported frustration about gaining weight, intending to place a third BIG, however it depended on their emotional and financial stability.

\section{Results}

In the period of the use of the second intragastric balloon, in February 2016 with 4 months of IGB use, the patient was found to be pregnant with her second child, when it was necessary to withdraw the IGB again, with a final weight of $65.5 \mathrm{~kg}$ and BMI of $25.92 \mathrm{~kg} / \mathrm{m}^{2}$. Thus, with the IGB insertion, there was an improvement of the BMI and hormonal levels, which favours increasingly the fertility and consequently the gestation. Among the most common side effects it is observed nauseas, vomiting, pain, abdominal discomfort, gastroesophageal reflux disease, halitosis, eructations and flatulence, as well as the satiety sensation observed in patients. After the second IGB was withdrawn, the patient did not continue with nutritional and psychological follow-up, so by April 2016 her weight was already $75.5 \mathrm{~kg}$, and in September 2016, at 39 weeks' gestation, her weight was $89 \mathrm{~kg}$. The patient reported frustration regarding weight gain, intending to place a third IGB, however it depended on her emotional stability. 


\section{Discussion}

The epidemic of overweight and obesity has become one of the biggest public health problems in the world and is increasingly common among women of childbearing age. The most probable cause for its onset is related to genetic factors and the predisposition of the individual to present a positive energy balance, which happens when the amount of energy consumed is greater than the amount spent in performing vital functions and activities in general. Grade I obesity is classified when BMI (body mass index) is equal to or greater than $30 \mathrm{~kg} / \mathrm{m}^{2}$, grade II when the BMI is equal to or greater than $35 \mathrm{~kg} / \mathrm{m}^{2}$ and grade III when the BMI is equal to $40 \mathrm{~kg} / \mathrm{m}^{2}$ or higher than this value [5].

Obesity is a disease that can interfere with sexuality, considering a chronic, metabolic and diverse etiology, whose incidence has been increasing in the last two decades, both in developed and developing countries. Increased body fat may alter the balance between the bioavailability of estrogens, androgens, and circulating sex hormones, leading to clinical manifestations and dysfunction of the hypothalamic-pituitary-gonadal axis. Obese people often have body image disorders and a strong impact on appearance the impact of obesity on reproductive function, especially ovulatory disorders, can be attributed mainly to the endocrine mechanisms that are able to reduce ovulation rates [5].

Obesity is very prevalent in women with PCOS (polycystic ovary syndrome), which is a common cause of infertility. PCOS affects 5 to $7 \%$ of women and is often associated with women with a body mass index $>$ $25 \mathrm{~kg} / \mathrm{m}^{2}$. Anovulation is more common in women with obese PCOS (>50\% of patients with PCOS) than in non-obese PCOS patients [6]. The endocrine profile that reflects anovulation in obese women is characterized by: high insulin and LH (luteinizing hormone), abnormal FSH (follicle-stimulating hormone), and low progesterone. Many obese women are hyperinsulinemic and, therefore, have endocrine profiles characteristic of PCOS, that is, peripheral insulin resistance leads to a reduction in SHBG (carrier globulin of sexual steroids). Thus, there will be a greater free fraction of estrogen and androgen, inhibiting the pulsating release of $\mathrm{GnRH}$, deregulating ovulation [3]. In response there is an increase in sex steroids like testosterone, dihydrotestosterone and androestenediol. There is a functional hyperandrogenism in these women and this can lead to an effect on ovarian pathophysiological function and contribute to menstrual dysregulation and oligo-anovulation. Insulin also acts on the ovaries by stimulating steroidogenesis in theca and granulosa cells, and enhances the stimulatory effect of $\mathrm{LH}$ through upregulation of the $\mathrm{LH}$ receptor and; in the pituitary can increase the sensitivity of the gonadotrophic cells to the action of GnRH, further increasing the stimulation to steroidogenesis. Obesity, especially central obesity, induces a state of insulin resistance and in hormonal response, there is hyperinsulinemia to compensate [7].

Leptin also interferes with the relationship between obesity and fertility. Being a key signaling protein, this hormone transmits information about the peripheral energy reserves to the hypothalamus and has metabolic and reproductive functions. Leptin receptors have been demonstrated in the hypothalamus and pituitary and have been related in the control of gonadotrophin secretion [8].

In addition, some studies suggest that increased rates of abortion and early pregnancy loss, which is very common in obese women, can be attributed to a reduction in oocyte quality, affecting the development of the embryo, leading to a decrease in implantation rate and abnormal development of the trophoblast [6].

However, when conventional treatments (diet, physical activity, behavioral and pharmacological therapies) fail to produce significant and lasting weight reduction in patients with some degree of obesity, other approaches become interesting [1].

The IGB is one of the strategies currently available for weight loss with significant results in a short period of time. The BIG is placed in the stomach using 
endoscopy and left for up to 6 months, when it should be removed due to durability [4]. Three intragastric balloon devices are currently approved by the FDA in the United States: Orbera Intragas Balloon System (Apollo Endosurgery Inc, Austin, TX, United States), ReShape ${ }^{\circledR}$ Integrated Dual Balloon System (ReShape Medical, Inc., San Clemente, CA, United States), and Obalon (Obalon ${ }^{\circledR}$ Therapeutics, Inc.). These devices are indicated for patients with Class 1 and 2 obesity (BMI $30-40 \mathrm{~kg} / \mathrm{m}^{2}$ ) [2].

Intragastric balloon systems operate on the principle of inducing an anatomical sensation of fullness secondary to the space they occupy in the stomach cavity. Consequently, post-procedure patients remain full for extended periods of time between meals. The Orbera Intragastric Balloon and the Reformed Integrated Double Balloon are placed in the gastric cavity through the mouth through a gastroscope. The Obalon balloon is swallowed by the patient through guided fluoroscopy and endoscopy is required to remove the balloon [2].

The GEB (Garren-Edwards Gastric Bubble) was the first FDA-approved IGB in the USA in 1985. The device was placed endoscopically filled with $220 \mathrm{~mL}$ of air, and removed after 3 months. The GEB was later withdrawn by the FDA in 1990, following reports of numerous complications. Adverse effects of BGE included fine-bowel obstruction, secondary to unplanned deflation, gastric ulcers with gastrointestinal bleeding and gastric perforation, mainly due to premature polyurethane deflation. Current IGB devices are delivered in the stomach to reduce gastric capacity and provide feeling of fullness. In single-flask devices, the IGB is filled with $400-700 \mathrm{~mL}$ saline solution, but the ReShape double flask can be filled to a combined volume of $900 \mathrm{~mL}$. The methylene blue dye is added to alert patients to balloon leakage or rupture by the appearance of a blue color in the urine. Typically, IGB devices are placed and removed endoscopically with sedation or anesthesia. After placement of the balloon, patients are placed on a liquid diet and use of antiemetics to relieve nausea and vomiting. The IGB devices are removed after 6 months as the risk of balloon deflation and the possible migration increases substantially there after [9].

The balloons in the ReShape Integrated Dual Balloon System have a fill volume of $750-900 \mathrm{~mL}$ and are designed to fit the natural shape of the stomach. This double balloon design reduces the potential for migration of the device from the stomach to the intestines if balloon deflation occurs, thereby reducing the risk of intestinal obstruction. Due methylene blue is injected into the saline solution present inside the flask, serving as an indicator of deflation of the flask by turning the patient's urine into blue-green. The Orbera system involves a flask containing $400-700 \mathrm{~mL}$ of saline solution. Studies and trials have shown low rates of deflation in this system, leading to minimal migration and obstruction. The Obalon flask is a gas filled balloon system that works using similar principles. It consists of up to 3 intragastric balloons placed in the first 3 months. The patient swallows the capsule of the balloon catheter, which also contains a radiopaque marker that helps confirm its position under the gastroesophageal junction with fluoroscopy or $\mathrm{X}$-rays. Once this is achieved, the catheter is used to inject gas (mixture of nitrogen-sulfur hexafluoride) into the flask. Each flask has a volume of approximately $250 \mathrm{~mL}$, totaling $750 \mathrm{~mL}$ with 3 flasks [2].

The IGB placement procedure is performed with the patient under mild sedation (no need for anesthesia) with introduction and filling guided by endoscopy and lasts for around 20 minutes as shown in Fig. 2. Although it is not an invasive procedure and does not require hospitalization, it is usually performed in a room fully prepared for this type of procedure. hospitalization, it is usually performed in a room fully prepared for this type of procedure. After placement, the balloon is filled with saline with contrast and dye. The patient is left with the balloon for 4 to 6 months when it is then withdrawn also by endosopy and sedation. 


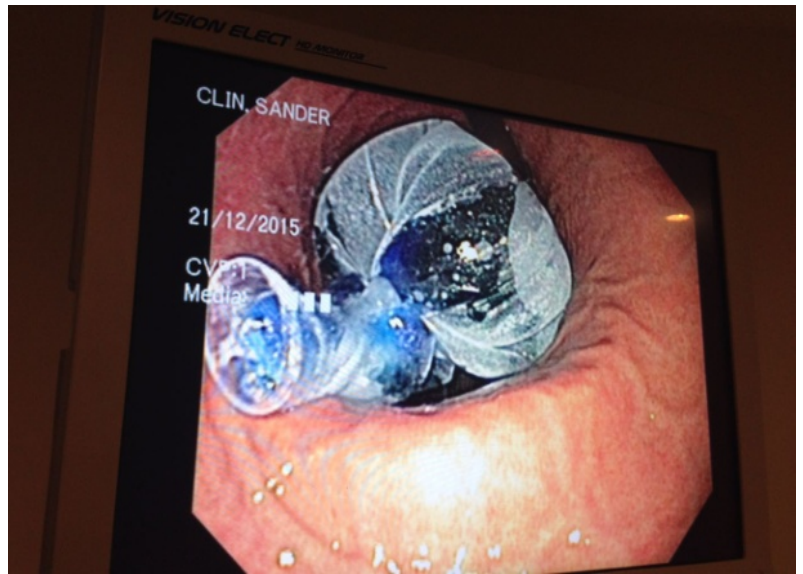

Fig. 2 Clínica sander.

In general, they do not present any restrictions to their routine physical and work activities, and their return to work is possible on the second day after the procedure [1].

For IGB placement the indications are: obese patients who rejected bariatric surgery; mobile obesity $(\mathrm{BMI}>40)$; patients with superobesity (BMI $>50)$ in preparation for bariatric surgery; obese patients with BMI between 35-40 with comorbidities in preparation for bariatric surgery; obese patients with BMI between 30-35 with chronic disease without solution; patients with BMI $<30$ associated with a multidisciplinary approach. The success of bariatric surgery and the intragastric balloon is due to the fact that it allows for greater weight loss and longer weight maintenance [3].

However, the technique has absolute contraindications, such as massive hiatal hernia, pharyngeal and esophageal anomalies, esophageal varices, use of anti-inflammatories or anticoagulants, pregnancy, pre-operated stomach and psychiatric disorders. Relative contraindications include grade III esophagitis, ulcer disease, and acute gastric mucosal lesion. Complications are related to the endoscopic method itself, sedation and balloon migration [10].

Although women need a certain minimum amount of body fat for reproductive efficiency in pregnancy, excess body fat can lead to menstrual irregularities, infertility, miscarriage, and difficulty performing assisted reproduction. There are numerous theories to explain the increased rate of abortion seen in obese women. Insulin resistance causes an altered release of progesterone, which may inhibit normal function of the corpus luteum. In addition, low levels of leptin may impair the early development of the embryo and impair the trophoblast invasion [3].

Obesity has been identified as a factor with a strong relation with infertility, and there are complex pathophysiological processes that are the basis of this association. Infertility is a prevalent problem throughout the world and entails not only significant psychosocial problems economic costs to society. Obese women are three times more likely to become infertile than a normal-weight woman. Most are not naturally infertile, but end up becoming due to the negative effects of obesity [11].

Obesity also impairs endometrial receptivity in embryo implantation and growth [3]. In addition to impairing spontaneous conception, a high BMI (body mass index) may impair the probability of reaching pregnancy with assisted reproductive technology [12].

Obesity in women who are in the reproductive period is difficult to manage. Diet, behavioral therapy, exercise and drug therapy are traditionally used, but they do not always work and it is common to recover from previous weight. In cases where there is a failure of several therapies, bariatric surgery may be indicated for the treatment of severe obesity, since it causes reduction of the subcutaneous fat, with consequent improvement of the peripheral resistance to insulin, causing improvement of the fertility, reduction of obstetric complications and neonatal morbidity. The American College of Gynecology and Obstetrics indicated that weight loss is essential for conception and recognizes bariatric surgery as a treatment in pre-pregnancy obesity, however, the procedure should be within a 2-year interval between surgery and pregnancy [3].

In view of this, intragastric balloon treatment is less invasive and has a lower complication rate than surgery and promotes a weight loss of five to nine points in the 
BMI at 6 months, as well as an impressive improvement in comorbidities associated with obesity in all the patients in whom surgery is not indicated for different reasons [3].

Therefore, weight loss has been successful in reducing insulin resistance and restoration of ovulation and fertility. This should be promoted as an initial treatment for obese women with infertility. Weight loss before conception is the ideal way to decrease the risk of medical and obstetric complications in obese women of reproductive age [13].

In the case report, the patient V.A.S.S, 38 years old, had an indication to place the BIG, since her BMI was $34.02 \mathrm{k} / \mathrm{m}^{2}$. In addition, she was undergoing hormone therapy to try to get pregnant for about 3 years and did not use contraceptive methods, so the couple was considered infertile, whereas one of the possible causes for this was the obesity of the patient V.A.S.S. BIG therapy was indicated by the gynecologist of the patient as a way to try to lose weight and thus achieve fertility. Reduction of fertility in obese women occurs due to an endocrinopathy characterized by: high insulin and LH (luteinizing hormone), abnormal FSH (follicle-stimulating hormone), and low progesterone. Many obese women are hyperinsulinemic and, therefore, have endocrine profiles characteristic of PCOS (polycystic ovary syndrome), that is, peripheral insulin resistance leads to a reduction in SHBG (sex-steroidal carrier globulin). Thus, there will be greater free fraction of estrogen and androgen, inhibiting the pulsating release of $\mathrm{GnRH}$, deregulating ovulation. Insulin resistance also causes an altered release of progesterone, which may inhibit normal function of the corpus luteum. In addition, research suggests that leptin deficiency may be an independent cause of infertility in obese women, since leptin receptors have been identified in the ovaries and testes, indicating that leptin has action on the functioning of the reproductive glands. Thus, low levels of leptin may impair the early development of the embryo and impair the invasion of trophoblast. Obesity also impairs the endometrial receptivity regarding the implantation and growth of the embryo. After 4 months with BIG, a patient with BMI of $25.91 \mathrm{~kg} / \mathrm{m}^{2}$ was able to become pregnant. This probably happened due to weight loss, causing reduced insulin resistance. With this, a normalization of SHBG occurred, reducing the free estrogen and androgen fractions and, thus, allowing the pulsating release of $\mathrm{GnRH}$, with consequent regularization of ovulation. In addition, it is important to emphasize that not only was there an increase in fertility due to weight loss, but also an improvement in the patient's self-esteem, enabling a more active sexual life, which also contributed to pregnancy.

\section{Conclusions}

In the face of the case report and after an accurate search in the literature, it was concluded that weight loss actually favors fertility, but self-esteem may have been determinant to improve sexual life, allowing conception. In both hypotheses the intragastric balloon was decisive for weight loss with increased fertility and improved sexual life.

\section{Conflicts of Interest}

There are no conflicts of interest.

\section{References}

[1] Comparotto, H., et al. 2014. "Balão Intragástrico: Evolução do Tratamento Clínico Multidisciplinar na Perda de Peso e Circunferência Abdominal." Revista Brasileira de Obesidade, Nutrição e Emagrecimento 8 (45): 79-86.

[2] Vyas, D., Kaivalya, D., and Yagnik, P. 2017. "Advances in Endoscopic Balloon Therapy for Weight Loss and Its Limitations." World Journal of Gastroenterology 23.44 (2017): 7813-7.

[3] Musella, M., et al. 2011. "The Potential Role of Intragastric Balloon in the Treatment of Obese-Related Infertility: Personal Experience." Obesity Surgery 21 (4): 426-30.

[4] Guedes, E. P., et al. 2018. "Impact of 6 Months of Treatment with Intragastric Balloon on Body Fat and Quality of Life in Obese Individuals with Metabolic Syndrome." Health and Quality of Life Outcomes 15 (2017): 211.

[5] Giviziez, C. R. et al. 2016. "Obesity and Anovulatory 
Infertility: A Review.” JBRA Assist Reprod.

[6] Nogueira, A. I., and Carreiro, M. P. 2013. "Obesidade e gravidez.” Revista Médica de Minas Gerais. 23.1.

[7] Yarak, S., et al. 2005. "Hiperandrogenismo e pele: síndrome do ovário policístico e resistência periférica à insulina." Anais Brasileiros de Dermatologia 80.

[8] Romero, C. E. M., and Zanesco, A. 2006. "O papel dos hormônios leptina e grelina na gênese da obesidade." Revista de Nutrição 19 (1).

[9] Tate, C. M., and Geliebter, A. 2017. "Intragastric Balloon Treatment for Obesity: Review of Recent Studies." Adv
Ther.

[10] Centro Cochrane do Brasil. 2006. Balões Intragástricos para Pessoas com Obesidade. São Paulo.

[11] Leitão, P. P. S. 2012. "Impacto da Obesidade na Fertilidade Feminina. 2012. 34f. Dissertação de Mestrado Integrado em Medicina - Universidade do Porto, Porto.

[12] Bellver, J., et al. 2007. "Obesity and Poor Reproductive Outcome: The Potential Role of the Endometrium." Fertility and Sterility 88 (2): 446-451.

[13] Fernandes, M. A. P., et al. 2007. "Intragastricballoon for Obesity." The Cochrane Library. 